\title{
Towards Micro-Assembly of Hybrid MOEMS Components on Reconfigurable Silicon Free-Space Micro-Optical Bench
}

\author{
S Bargiel ${ }^{1}$, K Rabenorosoa ${ }^{2}, C$ Clévy ${ }^{2}, C$ Gorecki ${ }^{1}$ and P Lutz ${ }^{2}$ \\ FEMTO-ST Institute UMR CNRS 6174 - UFC/ENSMM/UTBM, \\ ${ }^{1}$ Micro Nano Sciences, and Systems department (MN2S) \\ ${ }^{2}$ Automatic Control and Micro-Mechatronic Systems department (AS2M) \\ 25000 Besançon - FR \\ E-mail: sylwester.bargiel@,femto-st.fr
}

\begin{abstract}
:
The 3D integration of hybrid chips is a viable approach for the micro-optical technologies to reduce the costs of assembly and packaging. In this paper a technology platform for the hybrid integration of MOEMS components on a reconfigurable free-space silicon micro-optical bench is presented. In this approach a desired optical component (e.g. micromirror, microlens) is integrated with removable and adjustable silicon holder which can be manipulated, aligned and fixed in the precisely etched rail of the silicon baseplate by use of robotic micro-assembly station. An active-based gripping system allows modification of the holder position on the baseplate with nanometre precision. The fabrication processes of the micromachined parts of the micro-optical bench, based on bulk micromachining of standard silicon wafer and SOI wafer, are described. The successful assembly of the holders, equipped with micromirror and refractive glass ball microlens, on the baseplate rail is demonstrated.
\end{abstract}

Short title: Micro-assembly of MOEMS on reconfigurable MOB

PACS numbers: $85.85 .+\mathrm{j} ; 87.85 \mathrm{Uv}$

Keywords: micro-assembly, MOEMS, micro-optical bench, MOB, hybrid integration, silicon micromachining

Submitted to: Journal of Micromechanics and Microengineering

Corresponding author: Sylwester Bargiel; Tel: +33 (0) 3816663 02; sylwester.bargiel@femto-st.fr 


\section{Introduction}

The micro-optical technologies have made a significant progress in the past two decades and have found widespread applications in many areas, including telecommunications, sensors and biomedical fields [1]. Some of these applications require high precision for optical alignment while others involve the precise movement of small optical parts to achieve advanced functionalities [2-5]. It is strongly desired to integrate various functional elements of micro-optical system, such as free-space micro-optical components (lenses, gratings, mirrors, prisms), guided wave components (fibers, waveguides), microactuators or optoelectronic devices (laser diodes, photodetectors), on a single chip with sub-micron position accuracy to build cost- and space-effective optical modules. An increasing complexity of such systems poses new challenges to the assembly technology. The conventional assembly techniques suitable for many of these emerging applications have not kept pace with this progress due to their high costs and negative influence on the micro-optical system performance.

Silicon free-space micro-optical bench (FS-MOB), on the other hand, has been proven to be a powerful approach for the integration of active and passive components of optical systems on a single silicon substrate. In the MOB, the optical signals are transmitted in plane of silicon substrate through vertically positioned micro-optical elements that can be directly fabricated on the substrate (monolithic integration) or inserted as a discrete elements into dedicated mounting slots (hybrid integration). In both cases, the assembly process is significantly simplified. The position accuracy is also improved since the optical system can be "prealigned" by an appropriate design of photolithographic masks. The MOB structures can be fabricated with excellent precision and uniformity taking advantage of the novel achievements in micromachining and MEMS technology.

Especially, the surface micromachining has opened up an exciting opportunity to integrate monolithically the diffractive micro-optics with micropositioners, micro-actuators and optoelectronic elements (photodetectors) on a single silicon chip using basically the same technology $[2,6]$. In this technology, the micromirrors, Fresnel microlenses, beam splitters etc. are first fabricated on hinged polysilicon supporting plates by a planar process and then raised into vertical position either manually or by use of integrated scratch drive actuators. Self-assembled micro-XYZ stages with nm-range position accuracy were also demonstrated [7]. The complex MOEMS devices, such as an optical disk pickup head [8], can be fabricated with only little assembly. The optimisation of surface-machined micro-optical elements is often challenging because of the low diffraction efficiency of diffractive elements (e.g. maximum $10 \%$ for binary-amplitude or $41 \%$ for binary-phase Fresnel lenses) [9]) and restricted choice of wavelengths. Although the optical structures of arbitrary shape can be fabricated during planar process, the intrinsic stress in thin polysilicon layer often deteriorates their optical performance. 
The bulk micromachining of single-crystal silicon, on the contrary, provides various threedimensional (3-D) structures with low intrinsic stress, high aspect ratio and excellent surface smoothness. It was demonstrated that the strategy of bulk-micromachined silicon MOB can be applied for accurate passive alignment of optical components such as optical fibers [10] and the fabrication of high quality mirror surface [11]. Different geometry suitable for MOB can be obtained by combination of wet or dry anisotropic and isotropic processes of silicon etching (e.g. wet $\mathrm{KOH}$ or TMAH etching, RIE, DRIE). Especially, the use of silicon-on-insulator (SOI) wafers and Deep Reactive Ion Etching (DRIE) process has significantly widened the flexibility of MOB design allowing the fabrication of its structure in the well-defined, customer-specified layer of silicon (i.e. device layer). Powerful potential of this method was demonstrated by fabrication of complex systems in a single Si layer containing U-shaped guiding structures for fibers, micro-optical elements monolithically integrated with electrostatic or electrothermal micro-actuators $[2,12,13]$. Optical benches have also been fabricated using the LIGA process $[14,15]$. More recently, Descour et al. [16] proposed the concept of micro-optical table (MOT) where refractive and diffractive optical components are assembled into micromachined slots in the MOT substrate.

Despite the impressive progress in MOB fabrication, the easy assembling and alignment of hybrid-integrated micro-optical components remains a crucial problem. When assembly practice requires a human operator to pick and place micro-components manually using high power microscopes and microtweezers, the assembly is tiresome, time consuming and causes lots of losses. The micromanipulation station, appropriate for the positioning of micro-optical components, can seriously enhance the development of MOB applications. Consequently, innovative computer-controlled, teleoperated and semi-automatic robotized micro-assembly methods must be developed to increase efficiency, reliability, and reduce the costs.

Robotic micro-assembly systems are being intensively developed for the last few years. The influence of various micro-level forces, such as adhesion, electrostatic forces, and surface tension, on the assembly process has been studied [17, 18]. Micro-assembly of MOEMS parts requires specific systems that have to work in a limited free space and requests advanced control of a gripping tool (i.e. microgrippers). The microgripper has to be capable of grasping a micropart, removing it from the substrate, reorienting, translating and joining it to another micropart or to the assembling slot in MOB. Gripping tools can be classified as passive or active according to their working principle.

Passive ones do not include micro-actuators and are generally based on small bending structures with locking mechanisms $[19,20]$. Both the gripping tool and the grasped component must precisely fit together which requires a dedicated design and a very fine control of the micromachining tolerances [21]. Once designed and fabricated, the tool can be easily used. However, the limitations in the microfabrication 
processes, the dedicated design and fast wear are serious obstacles for their use in automated pick and place series.

On the other hand, active gripping tools include one or several micro-actuators for advanced functionality which makes them, however, difficult to control (non linear behavior, lack of sensors, small free space, influence of environmental conditions) [22]. Recent advances in the positioning control have shown that the active microgrippers are able to perform the reversible pick and place process [23]. This feature makes them a versatile solution for micro-assembly of MOB that requires the grasp of a variety of components.

The goal of this work is to combine the architecture of reconfigurable silicon free-space microoptical bench (RFS-MOB) and assembly technique based on robotic micro-assembly. This approach permits to built dynamically-aligned, reconfigurable 3D photonic micro-optical systems with microlenses, beam splitters and mirrors, detectors and light sources, that have to be assembled on two-level silicon bench platform, equipped with bulk-micromachined set of rails. The micro-optical elements can be fabricated independently on a dedicated holder using various materials (glass, polymer), other than the bench substrate (silicon), and using optimised technologies (bulk or surface micromachining, sol-gel, replication techniques). Based on the active gripping principle, generic holders with spring-based snap connectors have been developed enabling the insertion, guiding and fixing of various micro-optical components on the silicon baseplate. Thus, the modification of component's position on the baseplate becomes possible, making easy the reconfiguration of the complex microsystem and reducing manufacturing costs. Section 2 describes the concept of RFS-MOB while Section 3 deals with the design and the description of technological processes developed for different components of micro-optical bench. Finally, Section 4 describes the robotic workstation and methodology of micro-assembly procedure, leading to the successful assembly of chosen micro-components on MOB.

\section{Concept of RFS-MOB micro-assembly}

The concept of RFS-MOB is based on the hybrid integration of different heterogeneous 3-D micro-optical components on universal bulk-micromachined silicon substrate by use of robotic microassembly. The individual components of two-level architecture of the MOB are shown in figure 1(a). RFS-MOB contains a silicon baseplate with a set of mechanical rails in which a removable and adjustable silicon holder can be mounted, allowing the user to attach the desired micro-optical component. Figure 1(b) illustrates the concept of assembly of the holder into the rail of the silicon baseplate. 
a)
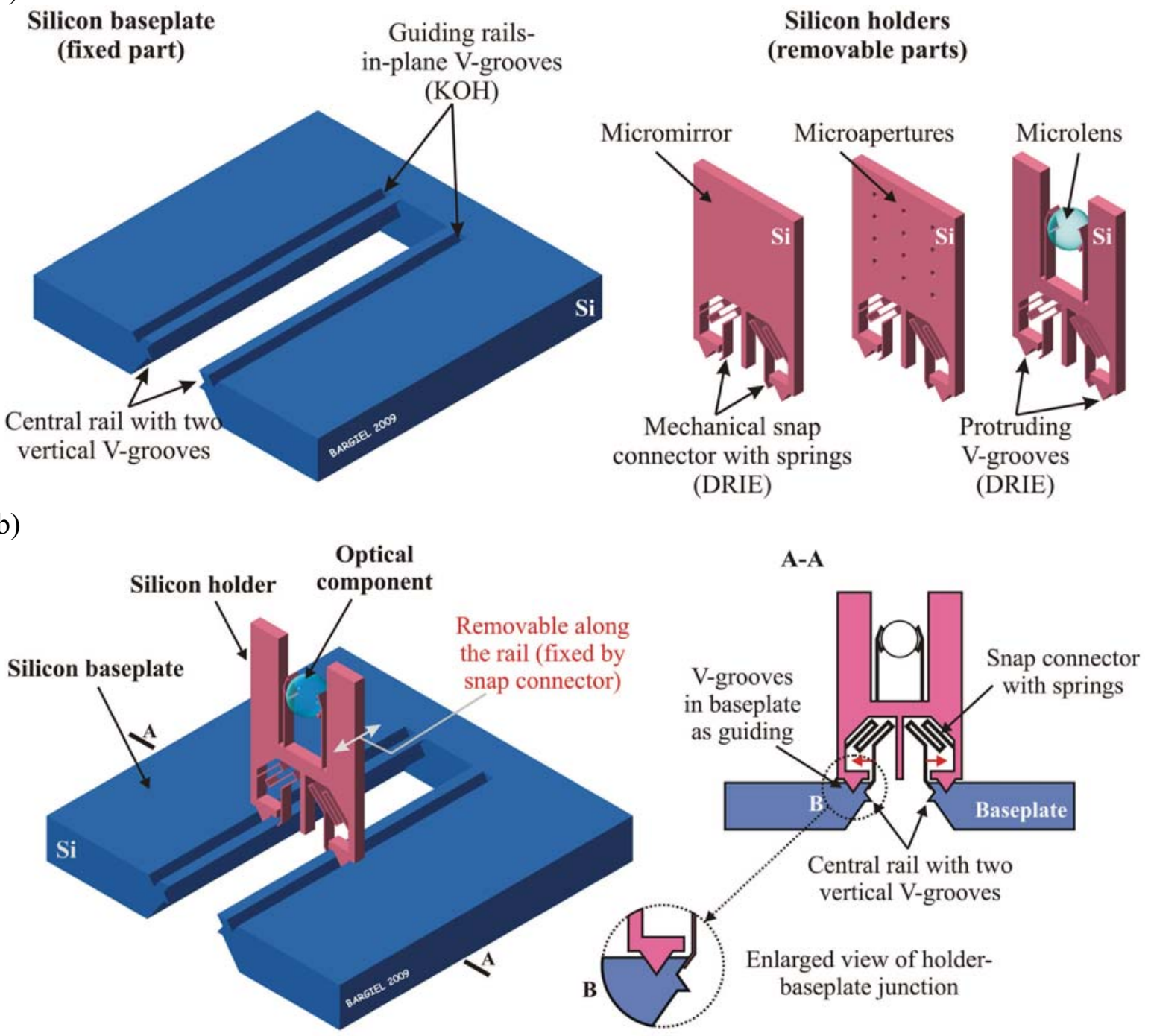

(DRIE)

\section{Silicon holders \\ (removable parts)}

V-grooves

(DRIE) b)

Figure 1. Individual components of RFS-MOB and their assembly concept: a) details of silicon bulk-micromachined baseplate and silicon holder (with ball microlens as micro-optical component), b) assembly of the holder into the central rail of the silicon baseplate.

All the operations concerning the manipulation of RFS-MOB elements (insertion, guiding, fixing, grasping, reorient) are made by use of active microgrippers. The modular design of the baseplate enables its alignment with the holder along $\mathrm{x}$-axis or $\mathrm{y}$-axis in the micromachined rails, allowing quick and coarse positioning of holder perpendicularly to the optical axis. The silicon baseplate constitutes the mechanical reference for various free-space micro-optical chips carried by the holder for precise alignment on micromachined rails along the optical axis.

The holder can be inserted into the straight rail of the baseplate as shown in figure 1(b). The alignment of the holder is obtained using a set of V-grooves produced by anisotropic etching of silicon: two standard in-plane V-grooves and two "vertical" ones. In-plane V-grooves are used as guiding rails, 
providing well defined surfaces of reference for other microparts of RFS-MOB - for instance, the holder is in contact with these rails through two protrusions with matched triangular cross-section, formed by DRIE. "Vertical" V-grooves enable mechanical fixing of the holder onto the baseplate. The components of RFS-MOB are fabricated separately by bulk-micromachining of standard silicon wafers (baseplate) or Silicon-On-Insulator (SOI) wafers (holder).

Figure 2 shows the steps of the general micro-assembly sequence, illustrating the insertion and displacement of holder within the rail of baseplate structure by use of microgripper. The main steps of this sequence are:

- picking up of the holder by active opening and closing motions of the microgripper's fingers and pre-positioning of the holder in front of the rail,

- insertion of the holder into the baseplate,

- guiding of the holder within the guiding rails to get the fine positioning of the holder,

- releasing of the snap connector springs through opening motion of the microgripper, which enables the fixing of the holder onto the baseplate,

- moving back motion of the microgripper.

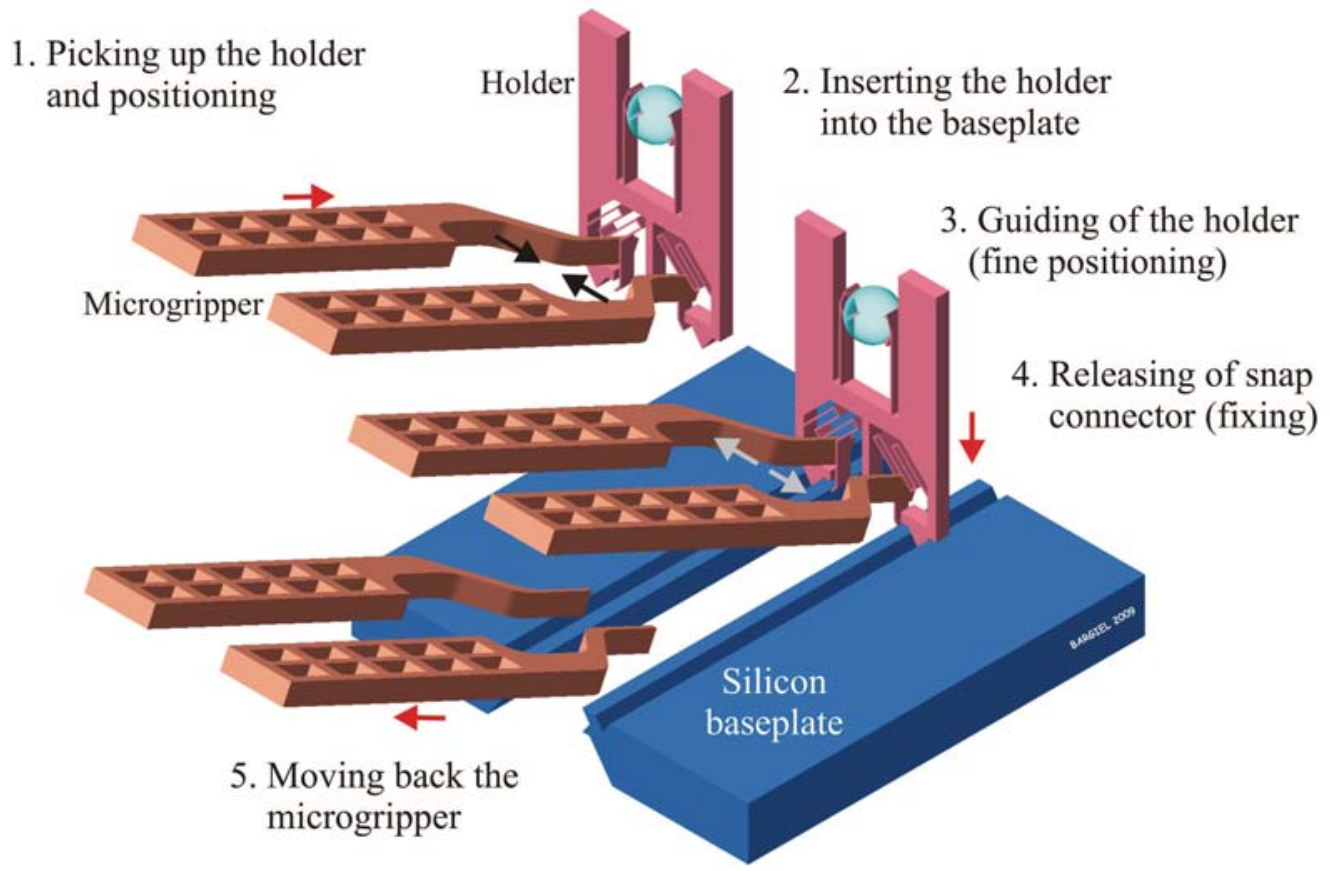

Figure 2. General concept of holder assembly based on the use of robotic active microgripper. The geometry of gripping tool, performing the insertion and displacement of holder within the rail of the baseplate, is illustrated. 
The control of the holder position is achieved by use of guide protrusions that match complementarily with the guiding rails in the baseplate. The locking system, implemented into the holder, contains a mechanical snap connector with two multi-folded springs. Once the springs are pressed by the microgripper, the holder can be inserted directly into the rail, adjusted in the optical setup, and then fastened by the releasing of the springs. After releasing, the tip of each spring is elastically adjusted to the surface of vertical V-groove.

One of the attractive features of the presented RFS-MOB technology is its ability to adjust actively the position of every optical part. Thus, the inaccuracy of the optical parameters coming from technological processes, e.g. deviation from the value of focal length of microlens, etc., can be compensated very precisely (motion resolution in the range of $10 \mathrm{~nm}$ ). This feature makes the RFS-MOB a flexible tool to build sophisticated batch-processed MOEMS devices at potentially low cost.

\section{Design and fabrication of silicon components of RFS-MOB}

\subsection{Silicon baseplate}

The technological investigations were first focused on the fabrication of rails for the silicon baseplate by use of the combination of standard processes of silicon bulk micromachining on standard (100)-oriented substrates. Wet anisotropic etching of silicon in aqueous solution of potassium hydroxide (KOH) was chosen to form crystallographically dependent 3-D structures, ensuring very good control of etch depth, excellent surface finish, and low cost. Using this method, the guiding rails (in-plane fabricated $\mathrm{V}$-grooves) can be attained in a simple one-step, self-stopped and self-centered etching process. However, the vertical V-grooves, required for locking of the holder on the baseplate, are more complex structures and their fabrication requires more effort.

One of the solutions concerning the fabrication of vertical V-grooves is based on local laser fusing of silicon, which damages its crystallographic structure, followed by the mask wet anisotropic etching [24]. Although desired shape of the structure with sidewalls bordered by two (111) walls on each side can be achieved in (100) oriented wafers, the laser fusing requires non-standard, dedicated equipment. In this work, an attractive alternative to this method was employed as a combination of two successive standard processes of anisotropic etching of silicon: dry deep reactive ion etching, followed by wet etching in aqueous $\mathrm{KOH}$ solution [25].

Figure 3 shows schematically the main technological steps of this process, applied to a test sample. First, the straight runner, aligned along to $<110>$ direction, was etched by DRIE in A601E machine (Alcatel, France) using 1.0- $\mu \mathrm{m}$-thick thermal $\mathrm{SiO}_{2}$ mask (steps 1-3). The structure was cleaned in oxygen plasma (step 4) in order to remove Teflon-like protecting polymer from the vertical sidewalls, deposited during 
DRIE process. In the second etching process, performed in $\mathrm{KOH}$ solution $\left(10 \mathrm{M}, 80^{\circ} \mathrm{C}\right)$ using the same $\mathrm{SiO}_{2}$ mask (step 5), each vertical sidewall was converted into two (111)-oriented sidewalls, inclined to each other at the angle of $\sim 110^{\circ}$. In the last step the $\mathrm{SiO}_{2}$ mask is stripped in buffered hydrofluoric acid (BHF) solution (step 6) and the cleaning of the final structure is performed.

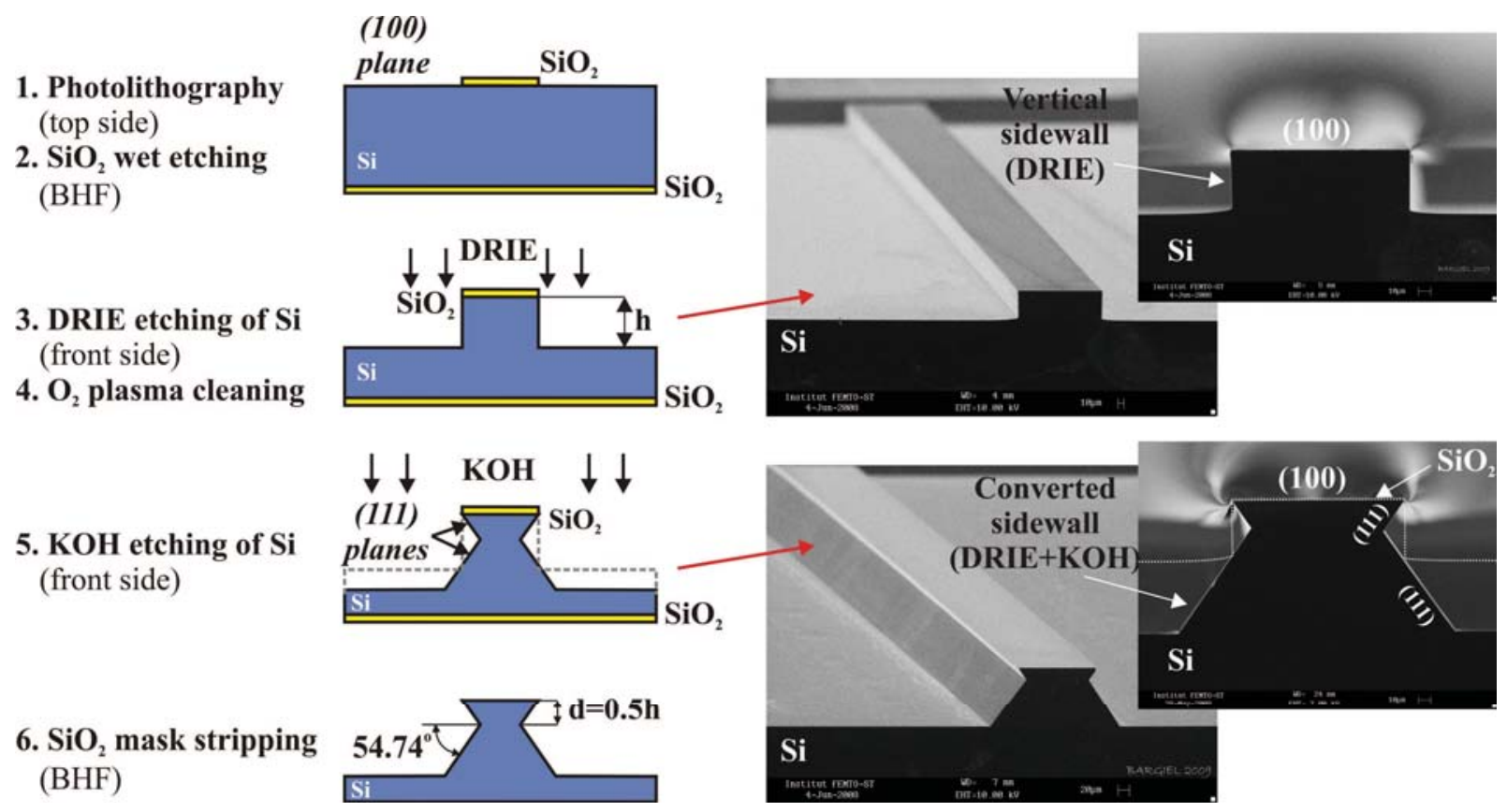

Figure 3. Fabrication of vertical V-grooves by the combination of two processes of anisotropic etching of silicon (dry DRIE followed by the wet etching in $\mathrm{KOH}$ ).

Tests have shown that this procedure is relatively simple and almost self-controlled. Nevertheless, some aspects have to be taken into consideration to control more precisely the dimensions of vertical Vgrooves as a part of micromachined rail. Especially, the distance $d$ between the wafer surface and the meeting point of two (111)-planes (figure 3 - step 6) has to be well controlled in order to ensure compatibility with the other RFS-MOB components. This distance is equal to the half of depth of the DRIE $(d=0.5 h)$, assuming an ideal profile of the vertical sidewalls (ideal vertical slop, flat bottom surface at the edge) and no underetching of $\mathrm{SiO}_{2}$ mask. Deviations from this ideal conditions result in the vertical shift of $d$ distance. For instance, in the test sample characterized by the negative sidewall slop of $1.7^{\circ}$ and typical slightly rounded profile at the bottom of $51 \mu \mathrm{m}$ deep cavity (figure 3, top SEM picture), the shift of this distance in the range of $\pm 2 \mu \mathrm{m}$ was observed. Although this inaccuracy is not big, it can be decreased by use of buried oxide layer, self-stopping the DRIE etching at the required depth, with the combination of low-frequency DRIE process, which ensures vertical sidewall slop at the surface of $\mathrm{SiO}_{2}$ layer. Such conditions of the etching can be achieved on SOI wafer or on the standard silicon wafer with 
wet anisotropically etched membrane, covered by thermal $\mathrm{SiO}_{2}$ layers. The latter was successfully employed in this work for the fabrication of the silicon baseplate, according the flow-chart presented in figure 4 .

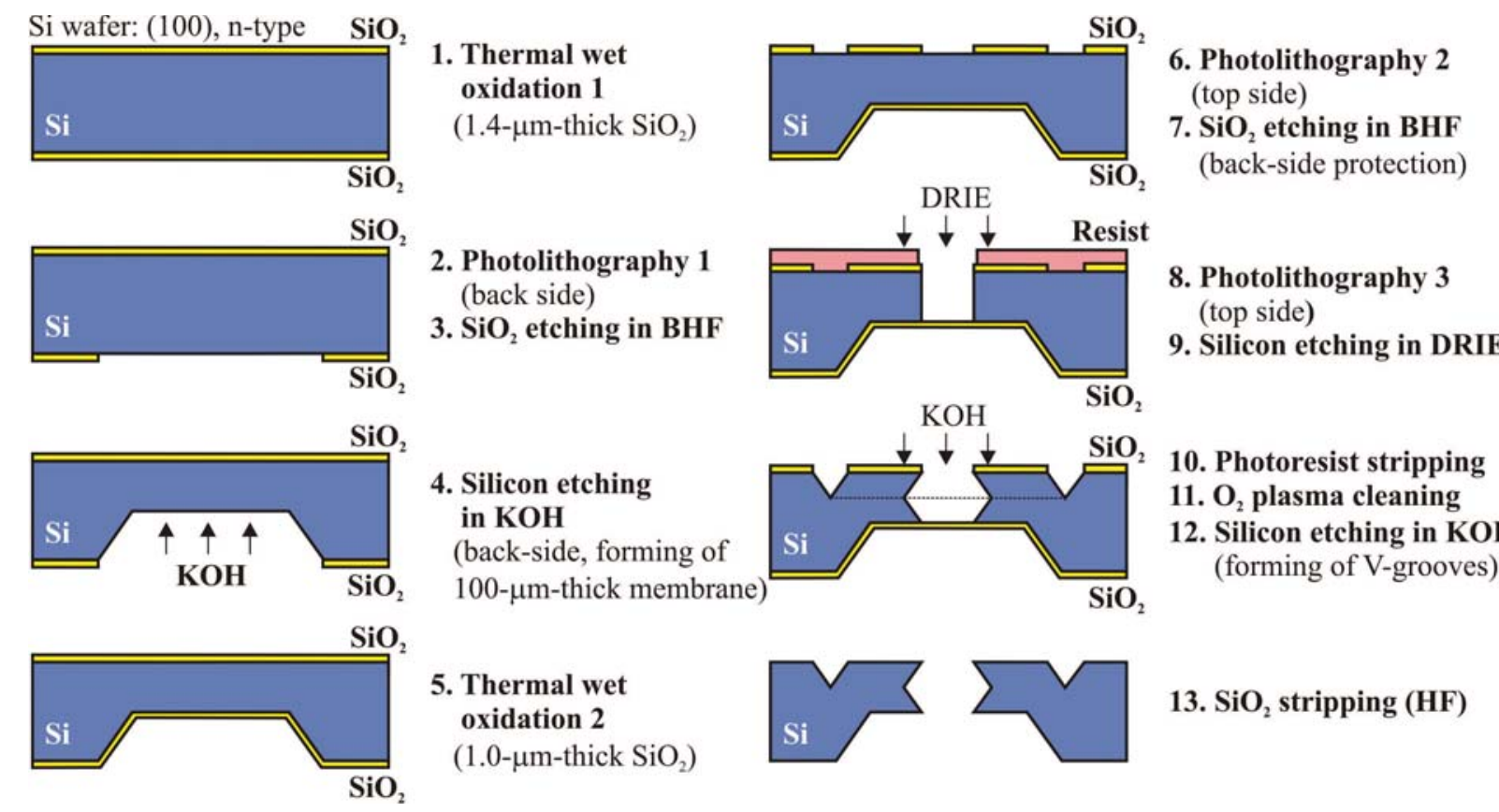

Figure 4. Technological flow-chart of the baseplate fabrication on the standard (100)-oriented silicon wafer.

The baseplate structures were fabricated on a n-type, (100)-oriented silicon wafer with thickness of $380 \mu \mathrm{m}$ and low total thickness variation (TTV $<3 \mu \mathrm{m}$ ). The wafer was wet thermally oxidized (step 1)

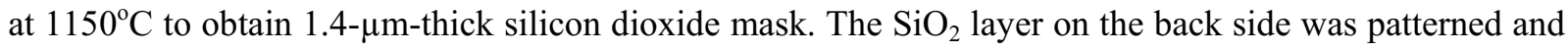
selectively etched in BHF solution (steps 2-3). Thereafter, the membranes were wet anisotropically etched in $10 \mathrm{M} \mathrm{KOH}$ solution at $80^{\circ} \mathrm{C}$ (step 4), followed by cleaning procedure and thermal oxidation of silicon $\left(1.0-\mu \mathrm{m}\right.$-thick $\left.\mathrm{SiO}_{2}\right)$ (step 5). The thickness of each membrane was carefully controlled to achieve $100-\mu \mathrm{m}$ thick and bilaterally oxidized layer of silicon for DRIE etching. In the next step, the top-side $\mathrm{SiO}_{2}$ layer was photolithographically patterned to obtain a mask for both DRIE and $\mathrm{KOH}$ etching (steps 6-7). The patterned $\mathrm{SiO}_{2}$ mask for $\mathrm{KOH}$ was selectively covered by SRP-220 (MEGAPOSIT ${ }^{\mathrm{TM}}$ ) photoresist before the DRIE of central rail (steps 8-9). The etching of rail self-stopped on the $\mathrm{SiO}_{2}$ membrane. After stripping of the photoresist and wafer cleaning, the $\mathrm{KOH}$ etching was performed in order to form simultaneously inplane V-grooves as well as vertical V-grooves. After stripping the $\mathrm{SiO}_{2}$ mask in $\mathrm{BOH}$ solution, the wafer was divided into individual chips by diamond saw dicing. The fabricated structure is shown in figure 5 . 
a)
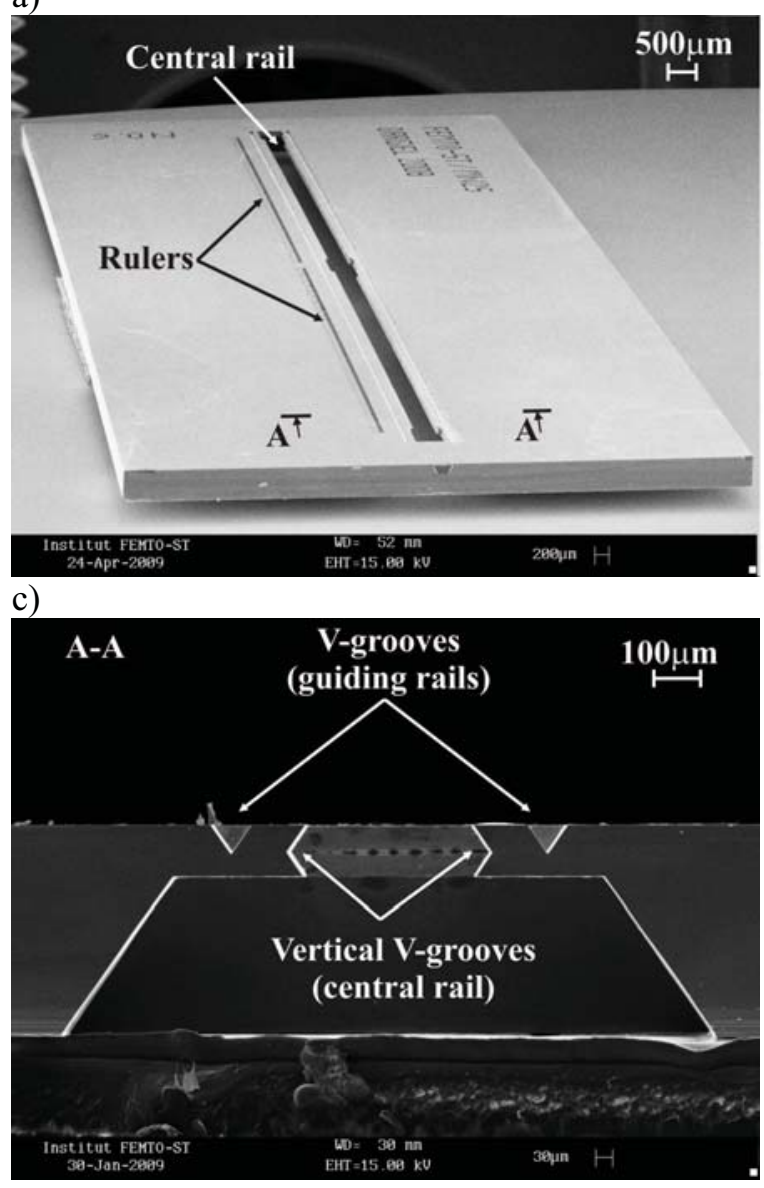

b)

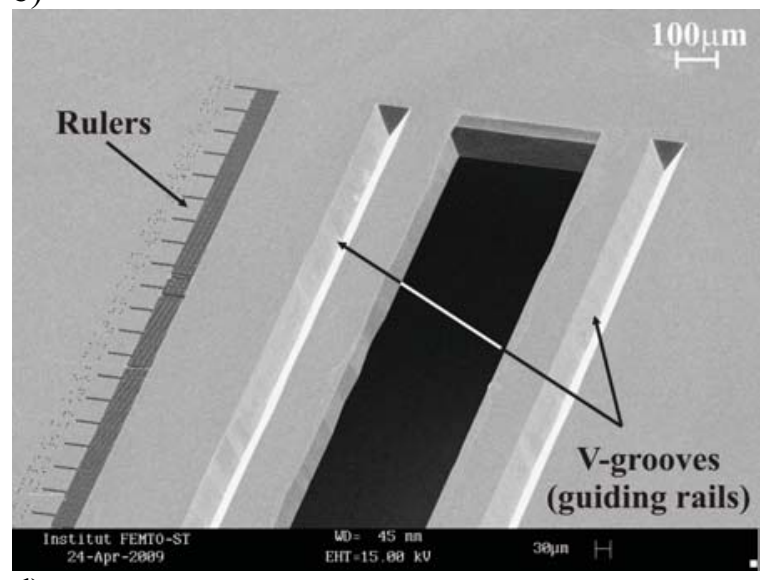

d)

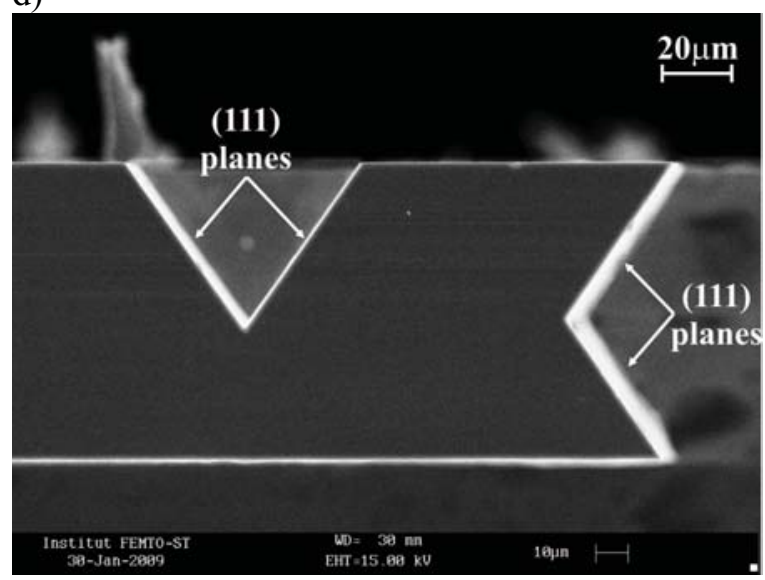

Figure 5. Example of silicon baseplate with one straight rail: a) general view, b) enlarged view of the 19-mm-long central rail, c) the cross-sectional view of the rail, d) enlarged view of the V-grooves: in-plane and vertical ones.

\subsection{Silicon holder}

The holder permits to align longitudinally and centre the micro-optical component along the optical axis as well as to lock it on the silicon baseplate by means of the integrated snap connector. As shown in figure 6 , the snap connector consists of a pair of springs with the width of $10 \mu \mathrm{m}$ and thickness of $50 \mu \mathrm{m}$, which are adjusted to the shape of central rail of the baseplate. 


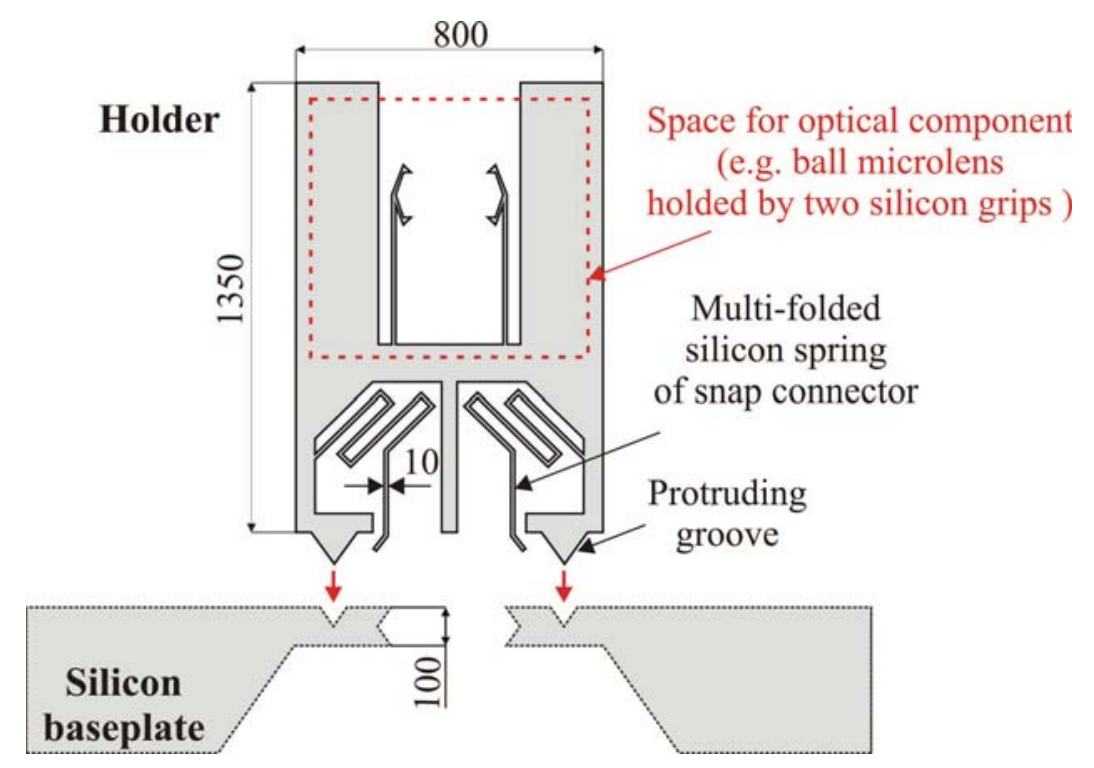

Figure 6. Construction of a silicon holder.

The protrusions with triangular cross-section ensure that the holder remains perpendicular to the optical axis during its sliding against the guiding rail of the baseplate. The locking of the holder into central rail of baseplate is achieved by generating the mechanical force (blocking force $F b$ ) between the tips of the springs and the top (111)-planes of vertical V-grooves. Since the locking step is crucial for assembly of RFS-MOB micro-parts, the most challenging part of holder design was to determine the appropriate shape and optimal mechanical properties of silicon springs. The spring construction had to be adjusted to the performance of the available piezoelectric microgripper [26, 27], characterized by the maximum gripping force of $55 \mathrm{mN}$, and to the required spring displacement of $130 \mu \mathrm{m}$. The size of the springs was another important design constrain since some working space had to be provided to allow assembly operation by the microgripper.

The multi-folded spring was chosen due to its robust and compact construction as well as low stiffness in the direction of movement. Mechanical properties of that spring were modelled by FEM using COMSOL software (figure 7). The aim was to obtain an optimal value of blocking force $F b$ which could guarantee the holder stability after assembly, but simultaneously, to ensure quasi-linear motion of the tip when it was deformed by microgripper with the gripping force $F g$. The latter condition was important in terms of stability of the grasping process. It was also noticed that the spring of the fixed holder was significantly bended. Therefore, the compensation of tips shape was required to improve their contact with the surface of vertical $\mathrm{V}$-grooves $\left(54.7^{\circ}\right.$ angle) . 


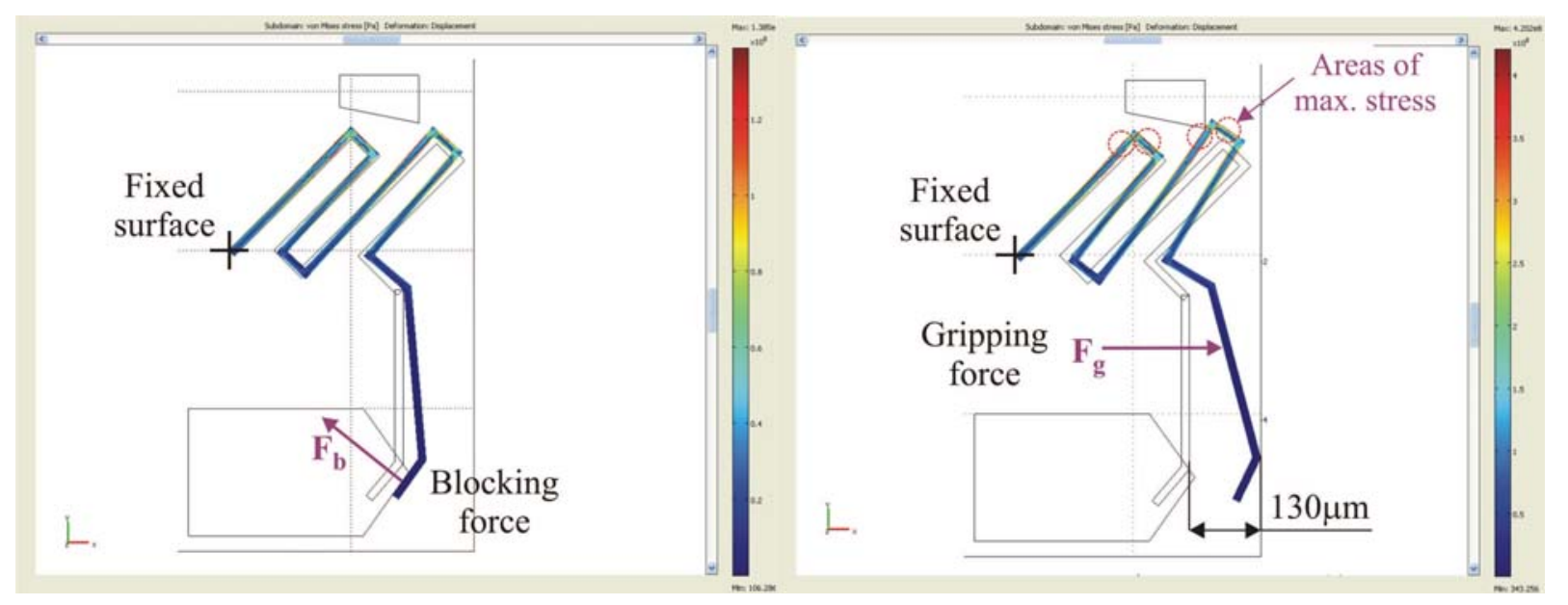

Figure 7. The results of FEM modelling (COMSOL software) of the multi-folded silicon spring as a part of micromachined snap connector, integrated into holder.

According to the results of FEM modelling, the optimum spring stiffness was estimated to be approximately $40 \mathrm{~N} / \mathrm{m}$ for the $10-\mu \mathrm{m}$-wide spring. The maximum values of von Mises stress ranging from $125 \mathrm{MPa}$ (blocking) to $420 \mathrm{MPa}$ (gripping) were obtained which is below the fracture strength of monocrystalline silicon in the microscale ( $1 \mathrm{GPa})$ [28]. The relatively small force of $F g=2.6 \mathrm{mN}$ has to be applied by the microgripper in order to grip the holder. When the holder is released into the central rail of the baseplate, each spring generates the blocking force of $2 \mathrm{mN}$.

The flow-chart of fabrication process of the holder is schematically shown in figure 8 . The holders were fabricated in a 50- $\mu \mathrm{m}$-thick device layer of 4", n-type and (100)-oriented SOI wafer with 1- $\mu \mathrm{m}$-thick buried oxide layer (BOX) and $400-\mu \mathrm{m}$-thick substrate layer. First, the 1.2- $\mu \mathrm{m}$-thick thermal $\mathrm{SiO}_{2}$ layer was created on the SOI wafer and subsequently patterned on both sides in the photolithography process using S1805 photoresist and BHF etching (steps 1-5). Then the holder structure was etched into the device layer by DRIE using A601E machine (Alcatel, France) (step 6). The springs with three different widths $(10 \mu \mathrm{m}, 12 \mu \mathrm{m}$ and $15 \mu \mathrm{m})$ were formed. In order to release the structures, a cavity was wet etched into the substrate layer in $10 \mathrm{M} \mathrm{KOH}$ solution at $60^{\circ} \mathrm{C}$. A chuck (AMMT, Germany) was employed to protect already formed silicon structures on the top side (step 7). Finally, the buried oxide was stripped in BHF solution, releasing the holder structure (step 8). At the end of the process the holders were maintained by tethers, described in [29]. 

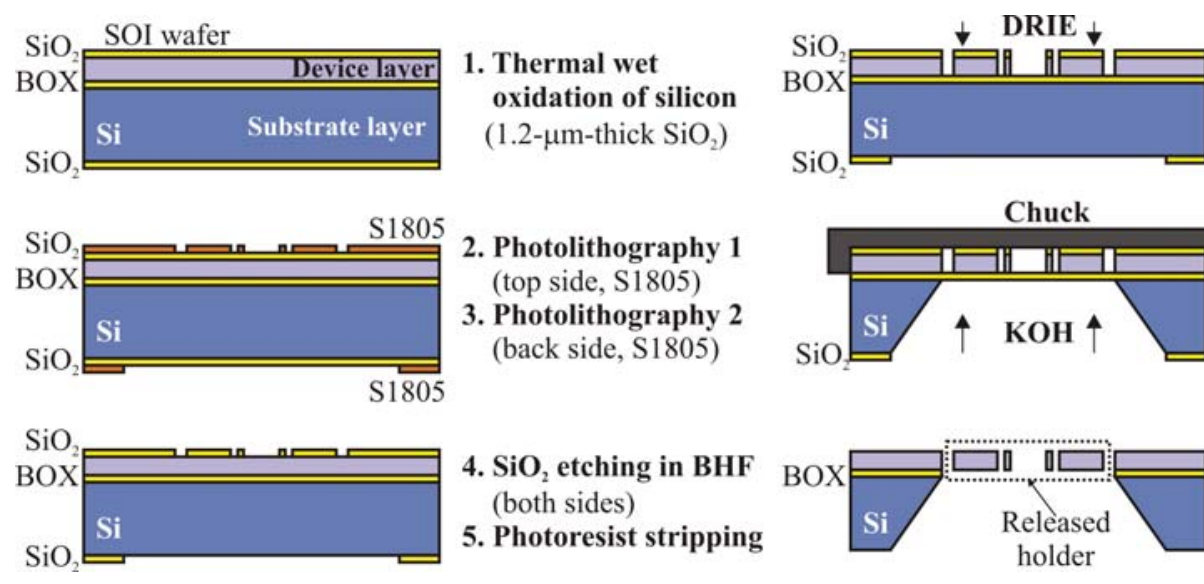

6. Silicon etching in DRIE

2. Photolithography 1 (top side, S1805)

3. Photolithography 2 (back side, S1805)

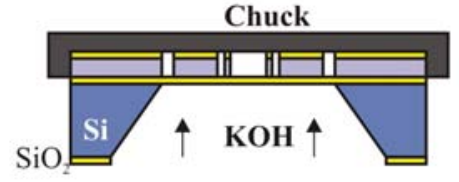

7. Silicon etching in $\mathrm{KOH}$ (back side, holder structure protected by chuck)

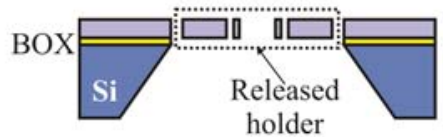

\section{8. $\mathrm{SiO}_{2}$ stripping in $\mathrm{BHF}$}

4. $\mathrm{SiO}_{2}$ etching in $\mathrm{BHF}$

(both sides)

5. Photoresist stripping

Figure 8. Flow-chart of the technological process of the silicon holder.

Due to the combination of SOI technology with DRIE process, it was possible to fabricate on the same SOI wafer several types of holders, already equipped with micro-optical components or prepared to be hybrid integrated with other micro-optical components, such as ball microlens. Figure 9 presents some examples of the fabricated holders containing: silicon micromirror (figure 9(a)), matrix of circular microapertures (figure 9(b)), silicon grips to hold the glass ball microlens of different diameter (figure 9(c)). All these components comprise the same deformable springs used to lock each holder on the baseplate rail during assembly. Since the holders are fabricated in planar process, in a user-defined layer of silicon, many other application-oriented structures, metallic or dielectric layers, or even active devices can be implemented.

a)

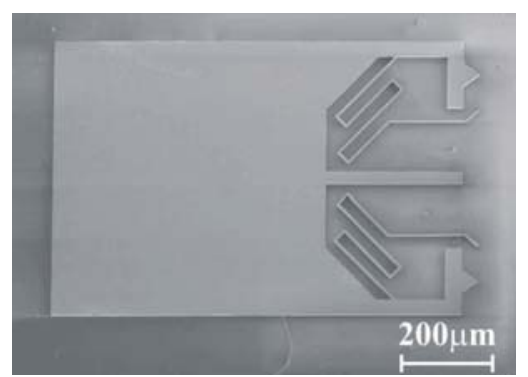

b)

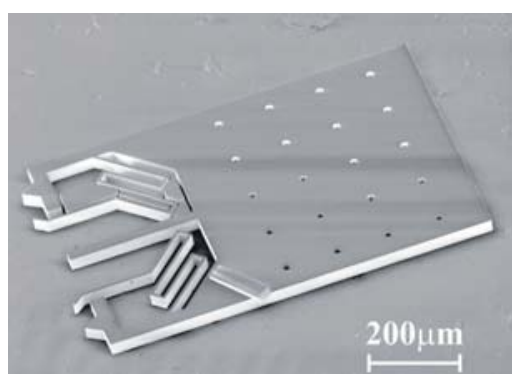

c)

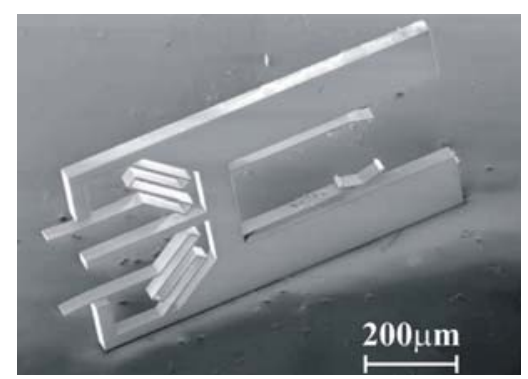

Figure 9. SEM pictures of released holders with different optical functionality: a) silicon mirror, b) matrix of circular apertures, c) silicon grips to hold the ball microlens. 


\section{Micro-assembly workcell and components assembly on RFS-MOB}

\subsection{Description of the micro-assembly workcell}

The workcell of the presented serial assembly system contains a robotic structure, a vision system and microgrippers performing tele-operated tasks. The role of the robotic structure is to generate a relative positioning of the manipulated holder and the baseplate. Motion in the range of several tens of millimetres must be ensured to pick a component from a wafer and to carry it to the baseplate. Thus XYZ macrorobotized stages are used to generate displacements at the "macro" scale. Because these stages do not generate motions with enough accuracy, XYZ fine positioning stages are used. They enable $100-\mu \mathrm{m}$-range motion with a resolution in the nanometer range. Finally, two fine rotational stages are used, the first one enables to rotate a holder of $90^{\circ}$ when it is picked from a wafer (flat positioning) to a vertical position (before inserting it in the baseplate rail). The second rotational stage enables the orientation of the baseplate rail relative to the direction of the guiding of a holder. Thus, the 3-D microrobotic assembly system containing eight degrees of freedom (DOF) stages, arranged into two robotic manipulators shown in figure 10, has been designed and developed.

The manipulator $\mathrm{R} 1$ is a large space positioning robot with four DOF $\left(\mathrm{x}_{1}, \mathrm{y}_{1}, \mathrm{z}_{1}\right.$ and $\left.\Theta\right)$. It is composed of linear coarse positioning stages from Physik Instrumente ${ }^{\circledR}$ - M112.1 DG (with $25 \mathrm{~mm}$ of travel range) and a rotation stage SmarAct ${ }^{\circledR}$ - SR-3610-S (with $1.1 \mu^{\circ}$ of resolution). The robot permits to break the tethers, pick, move, align to the groove and guide the holder. The manipulator R2 constitutes a work plan and it is composed of fine positioning robot with four DOF $\left(\mathrm{x}_{2}, \mathrm{y}_{2}, \mathrm{z}_{2}\right.$ and $\left.\beta\right)$ based on P-611.3 NanoCube ${ }^{\circledR}$ XYZ Piezo Stage with nanometric resolution (with $100 \mu \mathrm{m}$ of travel range) and a rotation stage SmarAct ${ }^{\circledR}$-SR-3610-S $(\Theta)$. The robot carries the substrate during the micro-assembly process and corrects the trajectory during the guiding operation. All of these stages are closed loop controlled. 
a)

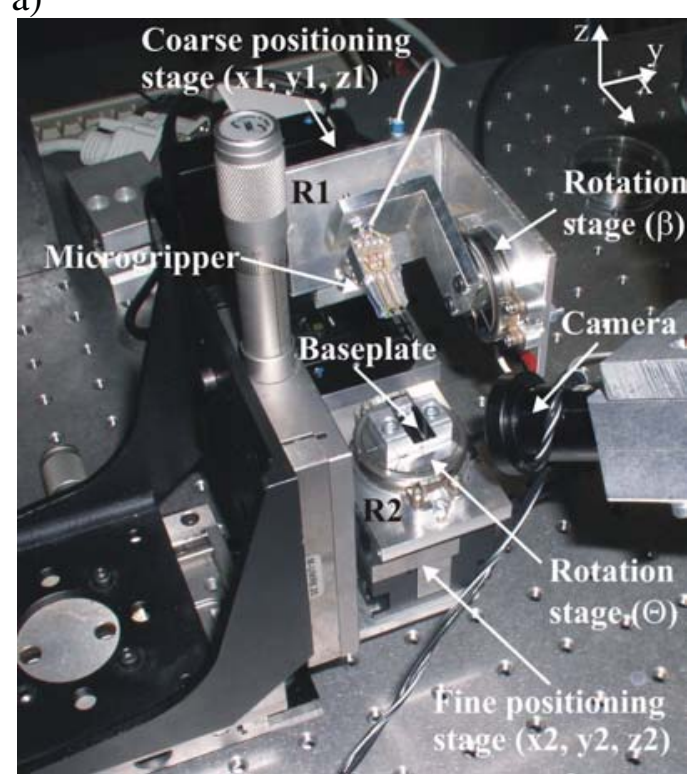

b)

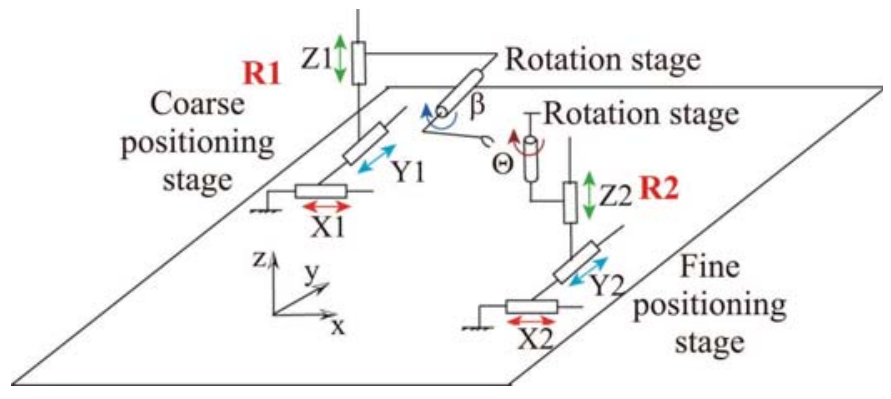

Figure 10. Configuration of the micro-assembly workcell with eight DOF: a) general view, b) kinetic view.

In order to grip the holder and ensure the reconfigurability of the RFS-MOB, a piezo-actuated microgripper MMOC (Micromanipulator-Microrobot-On-Chip), developed in our laboratory [30], was chosen. It has two active fingers and two DOF for each finger. Both fingers of the microgripper can move independently along y-axis and z-axis. It permits a stroke of $320 \mu \mathrm{m}$ in open-close motion (y-axis) and $400 \mu \mathrm{m}$ in up-down motion (z-axis). In reference to [31,32], the modularity of this microgripper has been largely proven. Appropriate end effectors (tools) can be chosen. The grasping is performed on the flexible part of the holder. The microgripper is mounted at the end of the manipulator R1.

The workcell is also equipped with a vision system. It helps with the alignment during inserting the holder into the rails, guiding, and supervising the whole assembly process. All of the eight motorized stages and the four DOF microgripper are controlled via a home made software based on $\mathrm{C}++$. It enables teleoperated assembly and automated pick and place tasks.

\subsection{Assembly process}

The described workcell configuration has been used to perform the micro-assembly process. In order to verify the concept of RFS-MOB, two different holders were chosen for assembly process: one carrying a glass ball microlens $(\phi=258 \mu \mathrm{m})$ and second with a silicon mirror. As ball microlenses, the soda-lime glass microspheres (Whitehouse Scientific, UK) with diameter of $(258 \pm 5.9) \mu \mathrm{m}$ have been employed, characterized by ultra-narrow size distribution, high sphericity, and long term stability. First, a microlens was inserted between the flexible springs (grips) of the holder using the microgripper. Then the 
holder with microlens as well as the holder with micromirror were grasped individually and inserted into the rail of the baseplate. Figure 11 presents the sequence of video snapshots which correspond to the main steps of the assembly process of holder with micromirror:

- picking, holding and $90^{\circ}$ angle rotation of the holder (figure 11(a)),

- generating motions to ensure a fine pre-positioning of the holder relatively to the baseplate plane which is used as a reference. The stability of the holder when grasped by the microgripper constitutes a critical issue requiring appropriate end effectors of the microgripper (figure 11(b)),

- guiding of the holder along the rail of the baseplate - fine motions enable the final fine positioning (figure 11(c)),

- releasing of the holder to lock it after the positioning (figure 11(d)).

a)

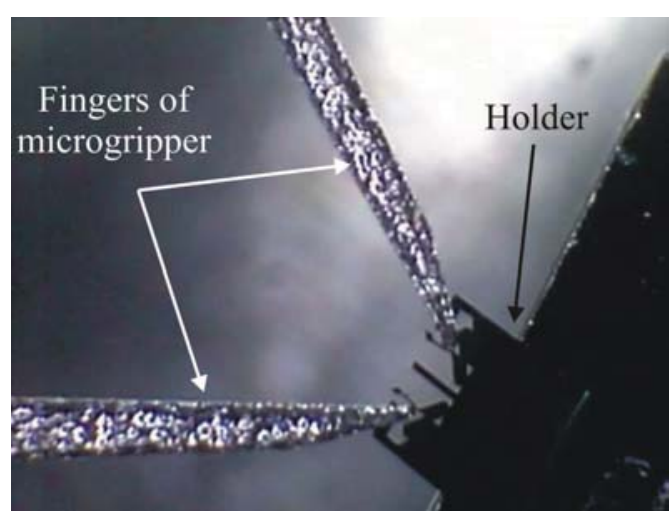

c)

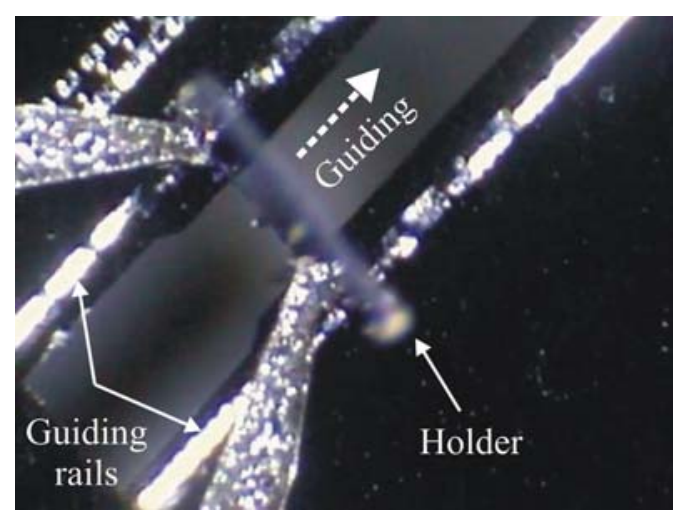

b)

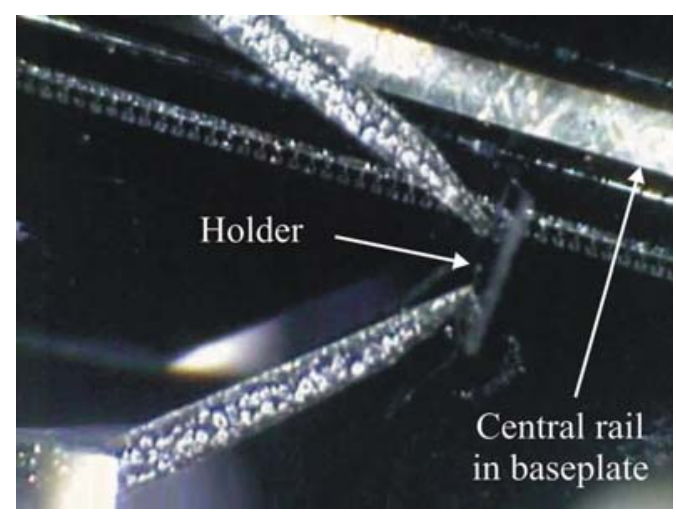

d)

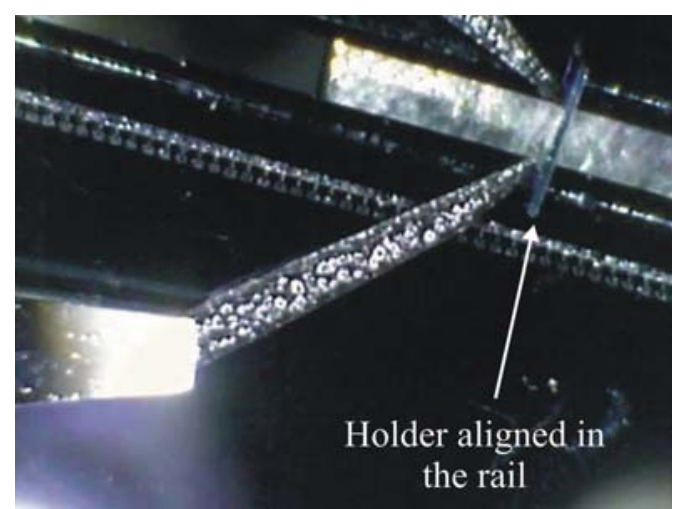

Figure 11. Sequence of the video snapshots showing the main steps of the holder assembly carried out by use of micro-robotic assembly station with piezoactuated microgripper: a) step 1: picking the holder on the flexible part, b) step 2: moving in space after 90 deg. rotation, c) step 3: guiding in the grooves along the rail, d) step 4: releasing.

The results of assembly process of RFS-MOT using a microgripper are shown in the figures 1213. Both holders were successfully assembled onto the baseplate which confirmed the compatibility of the holder construction with the construction of mechanical rails in baseplate (figure 12 (a)). 
a)

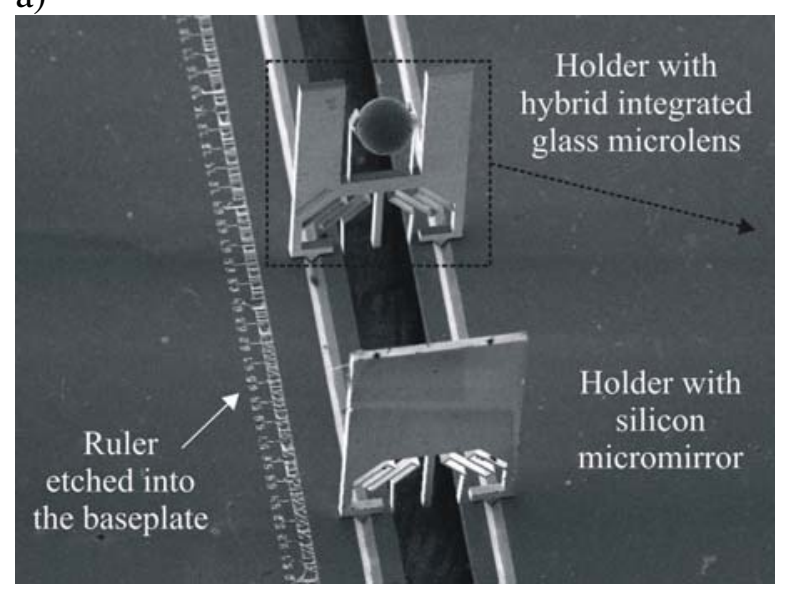

b)

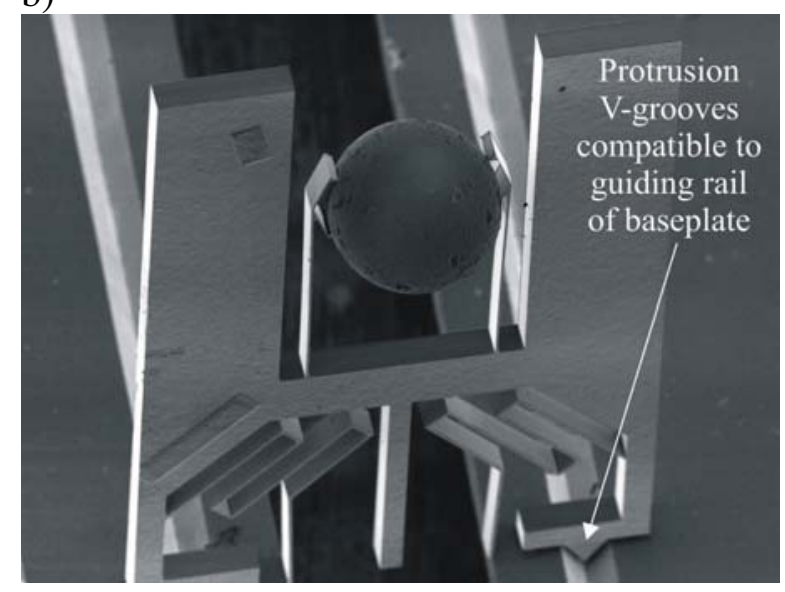

Figure 12. SEM picture of two holders assembled onto the rail of baseplate: a) general view, b) zoom on the central part of the holder carrying a ball microlens of $258 \mu \mathrm{m}$ in diameter.

The relative position of holders was controlled by use of the small scale (ruler), etched anisotropically into the baseplate on the left side of the components. Two silicon grips ensured stable holding and locking of the glass microlens during the whole assembly process (figure 12 (b)). Similarly, the snap connector has proved its usefulness for RFS-MOB as a universal locking mechanism for different micro-parts. Enlarged view of the holder-baseplate junction can be seen in figure 13.

The remarkable advantage of RFS-MOB is the fact that each component can be assembled anywhere along the central rail of baseplate which length can vary from hundreds of micrometers up to several centimetres. In practice, the maximum distance between holders is limited to the movement range of manipulators in micro-assembly station. On the other hand, already assembled holder can be replaced by a new one or adjusted by the microgripper which is capable of displacing of the object with the resolution in the range of $10 \mathrm{~nm}$.

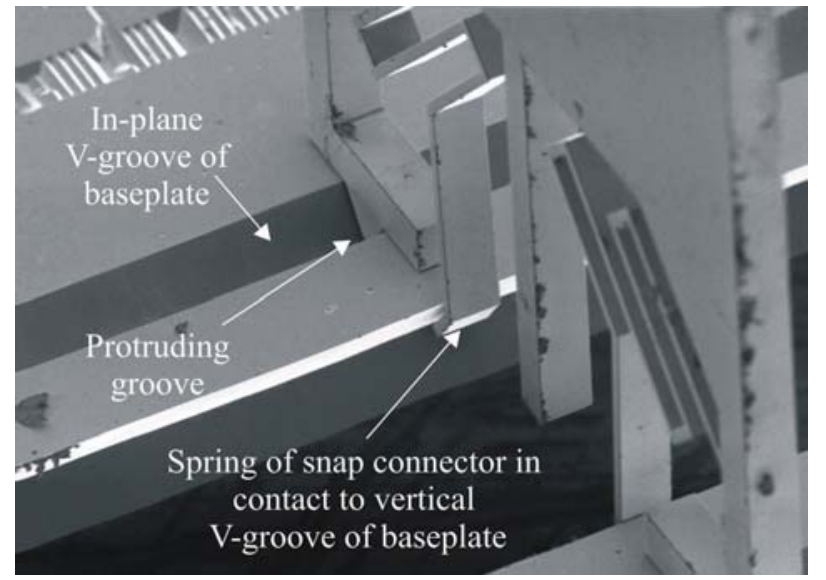

Figure 13. SEM picture of the holder-baseplate junction with magnification of sloped sides of protruding rail. 


\section{Conclusion and perspectives}

In this paper the concept of a reconfigurable silicon free-space micro-optical bench (RFS-MOB) was presented to bring a response to a new generation of complex MOEMS devices. This concept enables the development of new micro-optical systems based on the micro-assembly of holders with desired optical features on the universal silicon substrate (baseplate).

The design and the microfabrication of the different RFS-MOB components were presented. Original shapes obtained by bulk micromachining of silicon were used for guiding and locking the holders on the baseplate. The use of actuated microgripper ensured the reconfigurability of the micro-bench. The microassembly robotic system with eight degrees of freedom was developed to allow precise positioning of the components with nanometric resolution. The concept of RFS-MOB was successfully validated by assembly of different holders with hybrid and monolithic integrated optical components (microlens and silicon mirror) onto the baseplate in tele-operated mode.

The main characteristic of the developed micro-objects is their compliance. As a consequence, the control of accurate positioning is not achievable without a position sensor implemented into the microgripper. The fragility of flexible part has to be also taken into account. The use of the force sensor constitutes a promising solution. The integration of force sensor in the workcell is under current investigation. Force control will guarantee the stability of the grasp and will reduce the risk of breaking of flexible part. A hybrid force/position control will be applied in order to succeed automated assembly processes.

In future works the other type of optical components, such as diffractive microlens or beam splitters, will be developed according to the required RFS-MOB functionality. In addition, a new movable part of optical bench- silicon carrier, will be fabricated (figure 14) to introduce new adjustment possibilities for holders: in-plane rotation at discrete angles of 45 degree and free displacement along optical axis. In-plane rotation is very important for reflective microoptical components, e.g. micromirrors, allowing input/output of the light beam, optical interconnect, etc. In order to avoid disconnection of the carrier during its movement, a rhomboid-shaped runner (so-called "Z-lock" rail) will be formed on the carrier's back side, allowing in-plane, free movement in the base and simultaneously very limited movement in the vertical direction.

According to the reconfigurability of RFS-MOB, it can also be used as a tool for characterization of new micro-optical components and build proof-of-principle demonstrators. 


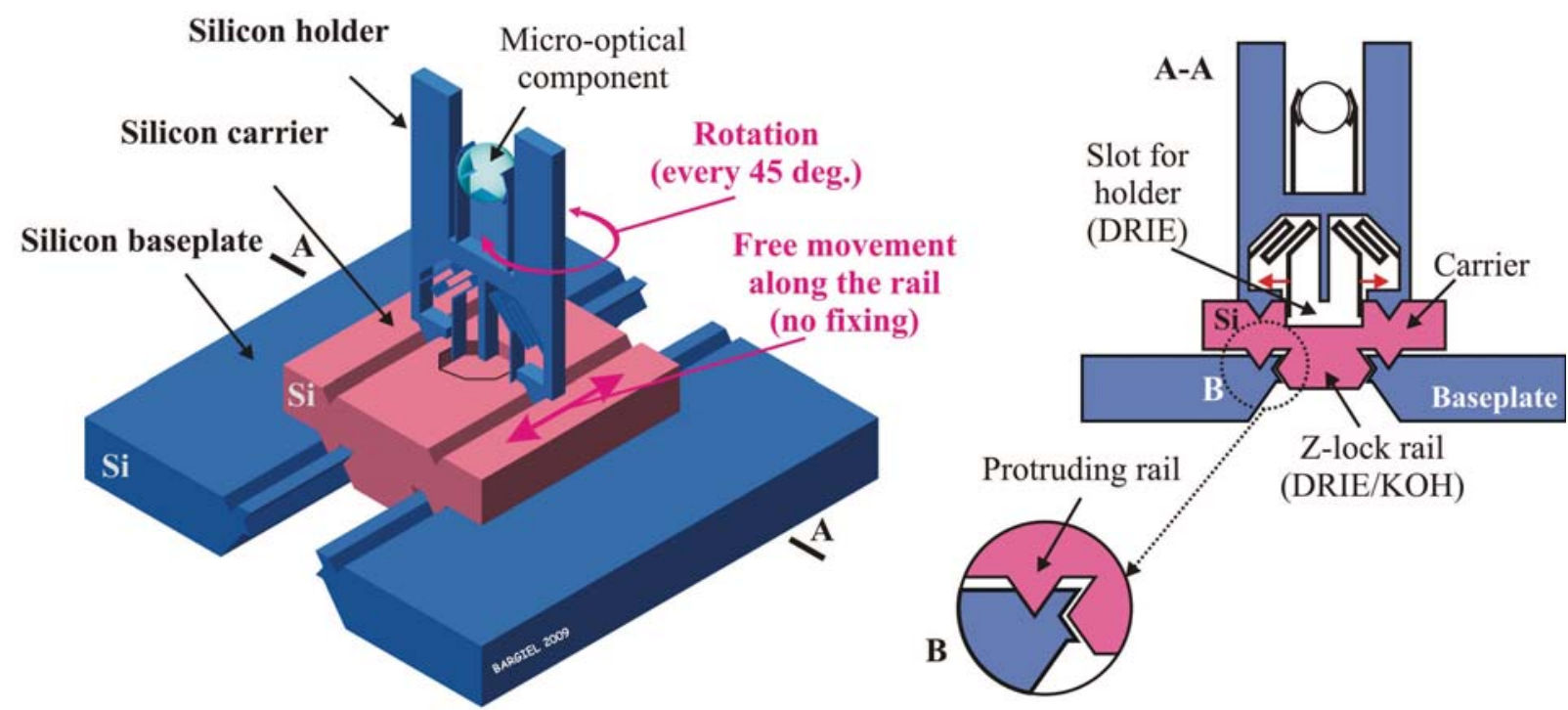

Figure 14. Towards new functionality of the RFS-MOB achieved by use of silicon carrier. Indirect assembly of the holder onto the carrier slot (left) allows its in-plane rotation and free movement along the rail whereas z-axis movement is very limited due to Z-lock rail (right).

\section{Acknowledgment}

The work was partially supported by the Franche-Comté region. The authors would like to thank David Hériban for his help in FEM modelling.

\section{References}

[1] Tolfree D and Jackson M J 2006 Commercializing Micro-Nanotechnology Products CRC Press

[2] Noell W, Sun W, Rooij N D, Herzig H P, Manzardo O, and Dändliker R 2002 Optical MEMS based on siliconon-insulator (SOI) for monolithic microoptics. Proc. 15th Annual Meeting of the IEEE Lasers and Electro-Optics Society vol 2, pp 580-581

[3] Chau F S, Du Y and Zhou G 2008 A micromachined stationary lamellar grating interferometer for Fourier transform spectroscopy J. Micromech. Microeng. 18 025-023

[4] Syms R R A, Zou H and Stagg J 2006 Micro-opto-electro-mechanical systems alignment stages with Vernier latch mechanisms J. Opt. A: Pure Appl. Opt. 8 305-S312

[5] Wolffenbuttel R F 2005 MEMS-based optical mini- and microspectrometers for the visible and infrared spectral range $J$. Micromech. Microeng. 15 145-152

[6] Syms R R A Optical 2005 MEMS in bonded silicon on insulator Design, Automation and Test in Europe (DATE'05), Messe, Munich

[7] Lin L Y, Shen J L, Lee S, Wu M C 1997 Surface-Micromachined Micro-XYZ Stages for Free-Space Microoptical Bench IEEE Photonics Technology Letters 9 No. 3 345-347

[8] Lin L Y, Shen J L, Lee S S and Wu M C 1996 Realization of novel monolithic free-space optical disk pickup heads by surface micromachining Optics Letters 21 155-157

[9] Rastani K, Marrakchi A, Habiby S F, Hubbard W M, Gilchrist H and Nahory R E 1991 Binary phase Fresnel lenses for generation of two-dimensional beam arrays Applied Optics 30 No. 11 1347-1354

[10] Aoki Y, Kato T, Mizuno R J, and Iga K 1999 Micro-optical Benach for alignment-free optical coupling Applied Optics 38 No. 6 963-965

[11] Uenishi Y, Tsugai, M, and Mehregany M 1995 Micro-opto-mechanical devices fabricated by anisotropic etching of (110) silicon J. Micromech. Microeng. 5 305-312 
[12] Hsieh J, Hsiao S Y, Lai C F, and Fang W 2007 Integration of a UV curable polymer lens and MUMPS structures on a SOI optical bench J. Micromech. Microeng. 17 1703-1709

[13] Yu K, Lee D, Krishnamoorthy U, Park N, Solgaard O 2006 Micromachined Fourier transform spectrometer on silicon optical bench platform Sensors and Actuators A 130-131523-530

[14] Ruzzu A C M, Haller D, Mohr J A, Wallrabe U 2003 Optoelectromechanical switch array with passively aligned free-space optical components J. Lightwave Technology 21 No. 3 664-671

[15] Khan Malek C and SaileV 2004 Applications of LIGA technology to precision manufacturing of high-aspectratio micro-components and -systems: A review J. of Microelectronics 35 131-143

[16] Descour M R, Kärkkäinen A H O, Rogers J D, and Liang C 2002 Toward the development of miniaturized imaging systems for detection of pre-cancer IEEE Journal of Quantum Electronics 38 No.2 122-130

[17] Gauthier M, Régnier S, Rougeot P, and Chaillet N 2006 Forces analysis for micromanipulations in dry and liquid media Journal of Micromechatronics 3 389-413

[18] Rabenorosoa K, Clévy C, Lutz P, Gauthier M and Rougeot P 2009 Measurement setup of pull-off force for planar contact at the microscale Micro-nano letters 4-3 148-154

[19] Das A N, Zhang P, Lee W H, Stephanou H, and Popa D $2007 \mu 3$ : Multiscale, deterministic micro-nano assembly system for construction of on-wafer microrobots Proc. IEEE International Conference on Robotics and Automation pp 461-466

[20] Dechev N, Cleghorn W L, and Mills J K 2004 Microassembly of 3-d microstructures using a compliant, passive microgripper Journal of Microelectromechanical Systems 13 176-189

[21] Popa D O 2009 High Yield Automated MEMS Assembly: Compliant Snap-Fastener Design, Precision

Robotics, and Assembly Evaluation to appear in Robotic Microassembly, ed. M. Gauthier, S. Régnier, IEEE Press

[22] Borovic B and Liu A Q and Popa D and Cai H and Lewis F L 2005 Open-loop versus closed-loop control of

MEMS devices: choices and issues J. Micromech. Microeng 15 1917-1924

[23] Rakotondrabe M, Clévy C and LutzP expected in 2010 Complete open loop control of hysteretic, creeped and oscillating piezoelectric cantilever IEEE - Transactions on Automation Science and Engineering (T-ASE), in press [24] Alavi M, Buttgenbach S, Schumacher A, Wagner H J 1992 Fabrication of microchannels by laser machining and anisotropic etching of silicon Sensors and Actuators A 32 299-302

[25] Saadaoui M, Pons P, Plana R, Bary L, Dubreuil P, Bourrier D, Vasilache D, Neculoiu D and Müller A, Dry followed by wet backside etching processes for micromachined endfire antennae $2005 \mathrm{~J}$. Micromech. Microeng. 15 $65-71$.

[26] Agnus J, De Lit P, Clévy C, and Chaillet N 2003 Description and performances of a four-degrees-of-freedom piezoelectric microgripper Proc. International Symposium on Assembly and Task Planning, pp 66-71

[27] Clévy C, Hubert A, Agnus J, and Chaillet N 2005 A micromanipulation cell including a tool changer. $J$. Micromech. Microeng. 15 292-301

[28] Yi T, Li L, Kim C-J, 2000 Microscale material testing of single crystalline silicon: process effects on surface morphology and tensile strength, Sensors and Actuators 83 172-178

[29] Hériban D, Agnus J, Petrini V, and Gauthier M 2009 A mechanical de-tethering technique for silicon MEMS etched with a DRIE process J. Micromech. Microeng. 19 1-9

[30] Perez R, Agnus J, Clévy C, Hubert A, and Chaillet N, 2005 Modelling, fabrication and validation of a high performance 2 DOF microgripper, ASME/IEEE Transaction on Mechatronics, 10(2) 161-171

[31] Nolan M Zyvex Labs 2008 Apparatus and methods of manufacturing and assembling microscale and nanoscale components and assemblies, US Patent No. 11131760

[32] Scussat M 2000 Assemblage bidimensionnel de composants optiques miniatures PhD thesis (In French), EPFL, Swizerland. 